\title{
FACTORES ORALES ASOCIADOS A HALITOSIS EN PACIENTES USUARIOS DE PRÓTESIS TOTAL QUE ASISTEN A LAS CLIÍNICAS ODONTOLÓGICAS DE LA UNIVERSIDAD SANTO TOMÁS
}

'Martha Lucia Rodriguez A., ' Jeonardo Moreno A. ' Carmen Alodia Marinez L. 'Estudiante de X semestre, F. de Odontologia. U. Santo Tomós. 'Odontòloga, U. Santo Tomós. Especialista en Ortopedio Maxilor, U. Antonio Nariño. Docente, U. Santo Fomás

Auror responsable de la correspondencia: Cormen Alodjo Martinez. $L$ correo plectrónico: aidolasz@hormail.com

Premio al mejor trabajo en el Área de Epidemiologia y al mejor trabajo de Pregrado en el XV Encuentro Nacional de Investigación Odontológica, ACFO. Universidad El Bosque, septiembre de 2004

\begin{abstract}
RESUMEN
Objetivo: Determinar lus factores orales usociados a halitosis en usuarias de prótesis total que asisten a las clinicas odontológicas de la Universidad Santo Tomás.

Materiales y métodos: Se realizó un estudio observacional analitica de corte transversal en una muestra de 102 pacientes de ambos géneros, entre 50 y 80 años, usuarios de prótesis totales superiores e inferiores y que estaban registrados en la base de datos de las clinicas odontológicas.

Resultados: El 69.6\% (71) fueron mujeres, el promedio de edad fue de $66.3+1-8.7$ nños. L.a halitosis a un palmo se relacionó con presencia de placa dentobacteriana en la superficie externa de la prótesis inferior con RR 2.88 , mal sabor en la boca con RR 2.16; consumo de ajo con RR 1.54; boco seca al levantarse con RR 1.48. La halitosis a un metro se relacionó con lesiones de mucosa labial con RR 7.84; lengua de color marrón con RR 19.6 y consumo de medicamentos (RR 2.95). No se encontraron pacientes con halitosis a tres metros.

Conclusiones: La halitosis a un palmo en pacientes usuarios de prótesis totales que acuden a la consulta odontológica a las clinicas de la Universidad Santo Tomás mostró que el mal sabor en la boca y la sensación de boca seca eran factores orales asociados a este fenómeno, asi mismo las lesiones en mucosa labial y la lengua color café podrían constituirse en factores bucales relacionados con la halitosis a un metro. [Rodriguez. ML. Moreno L. Martínez CA. Factores orales asociados a halitosis en pacientes usuarios de prótesis total que asisten a las clinicas odontológicas de la Universidad Santo Tombas. Ustasalud Odontnlogia 2005; 4: $9 \cdot 16$ ]
\end{abstract}

Palabras clave: Halitosis, Próresis totales, Medición organoléptica, Factores asociados.

\section{ASSOCIATED ORAL FACTORS TO HAIITOSIS IN PATIENTS WTTH DENTAL PROSTHESIS THAT ATTEND THE DENTAL CLINICS OF THE SANTO TOMAS UNIVERSTTY.}

\begin{abstract}
Objective: To determinate the oral factors associated with halitosis in patients with dental prosthesis who attended the Santo Tomns University dertal clinics.

Material and methods: An observational analytic cross-sectional study was done, with both gender-102 patients, with ages between 50-80 years old, rotal-denture wearers who were Santo Tomas University patients.

Results: The $69.6 \%$ of the patients were women: the age average was $66.3 .+1 \cdot 8.7$ años. Halitosis to a palm was related to dental biofilm across the external surfaces base mandibular dentadure with RR 2.88, oral bad taste with RR 2.16; garlic consuming with RR 1.54; xerostony during the early morning with RR 1.48. Halitosis to one meter was related with mucosa lips lesions with RR 7.84; brown tongue with RR 19.6 and medication consuming (RR 2.95). No patients were found with halitosis to three meters.

Conclusions: Holitosis to a palm in tolol-denture wearers who were Santo Tomos University dental clinic's patients was associated to intraoral factors related with bad oral taste and xerostomy. Halitosis to one meter was related oral factor how mucosa lips lesions and brown tongue.
\end{abstract}

Key words: Halitosis, Dental prosthesis, Organoleptic measures, Associated factors

Kecibida para publicacrón: 17 de abril de 2005. Aceptado para public ación: 20 de mayo de 2aw5. 


\section{INTRODUCCIÓN}

I. halitosis entendida como el olor bucal desagradable, está entre las condiciones médicas más comunes, aunque es relativamente poco lo que se conoce acerca de este embarazoso y en ocasiones frustrante problema.' Se constituye en una razón importante para visitar al odontólogo.?

En los últimos años se han logrado establecer las fuentes y las causas del mal aliento, pero sigue siendo un fenómeno de dificil diagnóstico y tratamiento por parte de los profesionales responsables de la salud oral. ${ }^{3}$

El olor bucal desagradable se puede producir por el consumo de ciertos alimentos o sustancias, enfermedad dental, periodontal, estomatitis, lengua saburral, carcinoma oral, fermentación de alimentos en la boca. xerostomía, Las prótesis dentales son una alternativa para aquellas personas parcial o totalmente edéntulas pues la sociedad de hoy exige que la ima. gen de la persona sea lo más agradable posible,' sin embargo los usuarios de prótesis pueden verse enfrentados a diferentes problemas asociados a éstas y que tienen que ver con la masticación, la fonación, la sonrisa y también, se ha asociado con la halitosis.

Factores relacionados con los materiales, el estado de la prótesis, su higiene y los factores retentivos se constituyen en elementos que podrian modificar las condiciones de halitosis en los pacientes usuarios de rehabilitaciones.

La halitosis, aunada a los otros problemas considerados en los usuarios de prótesis totales, puede arruinar una comunicacion de cualquier tipo, ser el origen de alteraciones psicológicas por parte del paciente, tendiendo al aislamiento social, evitando las relaciones familiares y/o conyugales e incluso llevar a la persona a problemas mentales y al aislamiento total. ${ }^{2 .}$

Las opciones de tratamiento son relativamente directas cuando el clínico puede, objetivamente, verificar la presencia de la halitosis y orientar las necesidades de tratamiento basados en un diagnóstico previo, fundamentado en la historia clínica y los factores asociados, con el fin de poder identificar los origenes de la halitosis, brindando a los pacientes posibles alternativas para disminuirla e incluso evitarla. ${ }^{21}$
Considerando lo expuesto, los usuarios de prótesis totales podrian registrar una serie de condiciones que podrian incrementar el riesgo a halitosis. Sin embargo, en la literatura revisada no se encontraron estudios que permitieran identificar con claridad, los factores orales asociados a este fenomeno en este grupo en particular. Por lo tanto, el objetivo del presente estudio fue determinar los factores orales asocindos a halitosis en usuarios de prótesis totales mayores de 50 años y que acuden a consulta de las clínicas de odontología de la Universidad Santo Tomás en Bucaramanga.

\section{MATERIALES Y MÉTODOS}

Se realizó un estudio observacional analitico de corte transversal de los factores orales asociados a halitosis en usuarios de prótesis total de 50 a 80 años, de ambos géneros, registrados en la base de datos de las clínicas odontológicas durante el año 2002, 2003 y primer trimestre del 2004 y que aceptaron mediante consentimiento informado participar en el estudio.

I.as especificaciones para la determinación del tamaño de muestra consideraron un nivel de confianza del $95 \%$, confianza del $80 \%$ una prevalencia de mula higiene oral en expuestos del 55\% y en no expuestos del 25\%, relación 1:1 y Riesgos Relativos: Razón de prevalencia (RR) 2.2 y considerado un $10 \%$ de pérdidas. De esta manera, se determinó que una muestra de 102 pacientes permitiría detectar diferencias estadisticamente significativas.

Las variables se consignaron en un instrumento diseñado para tal fin y se registraron de la siguiente manera:

Información general: consideró el registro de las variables sociodemográficas, asi como de aspectos relacionados sobre la percepción halitosis por parte del paciente. nivel de halitosis, presencia de mal sabor en la boca y consulta profesional por esta causa.

Hábitos dietéticos: se evaluo el tipo y la cantidad de alimentos como el ajo, la cebolla, los condimentos y el café.

Antecedentes médicos: se evaluaron las condiciones sistémicas del paciente en cuanto a presencia de enfermedades como: diabetes mellitus, sinusitis, problemas renales, enfermedades cardiovasculares, enfermedades gástricas, vómito, reflujo y consumo de algún medicamento.

Ustasalud Odontologia 2005; 4: 9 - 16

Rodriguez Ml. y col. 
Medidas de higiene oral: se evalủo los elementos de higiene oral utilizados para el lavado de la prótesis y la lengua.

Capa de la lengua: se examinó el color y el grosor de la lengua de acuerdo con una modificación de los criterios establecidos por Mantilla y colaboradores. ${ }^{5}$ La coloración fue clasificada en blanca, amarilla, café y negra. El grosor se determinó, sin cobertura, una cobertura delgada y una cobertura gruesa. Se consideró delgada cuando el color rosado subyacente se podía observar a través de la cobertura y gruesa cuando no era perceptible el color rosado bajo la cobertura.

Mucosa oral: se inspeccionaron los tejidos para buscar cambios de color, proliferación o destrucción de los tejidos bucales y se realizó palpación, aún cuando los tejidos blandos visualmente se observaran normales.

Estado protésico: se considero en las personas la necesidad de elaboración o cambio de prótesis de acuerdo con los criterios establecidos en el ENSAB III. ${ }^{6}$

Higiene de las prótesis: a nivel de las superficies dentales, considerando una modificación del ICP, pues se tomaron las superficies vestibulares de tres dientes artificiales en el maxilar superior y tres superficies linguales en el maxilar inferior lo que permitia obtener niveles de placa entre 0 y 3 . Se clasificó la higiene dental en buena, regular y mala. También se consideró la higiene de la base de las prótesis de acuerdo con los criterios establecidos por Pietrokovsky y colaboradores? que establecia el nivel de placa dentobacteriana considerando ninguna, poca, media y totol cobertura de placa en la superficie interna y externa de la prótesis superior y/o inferior.

Presencia de halitosis: se determinó la ausencia o presencia de halitosis mediante la prueba organoléptica a un palmo, un metro y tres metros.

Se efectuo una prueba piloto previa a la recolección de los datos. En ésta se evalúo el instrumento, los tiempos requeridos y la comprensión de las preguntas por parte del paciente. Además, fue necesario estandarizar a los examinadores para realizar la prueba organoléptica; este proceso se llevó a cabo mediante el apoyo de un examinador experimentado y finalmente, se procedió a la recolección de los datos de acuerdo con los criterios establecidos.

Los datos se procesaron en Fxcel y se exportaron al paquete Stata 8.0 para su correspondiente análisis. 3.9
El análisis de los datos se llevó a cabo de la siguiente manera: Para el análisis univariado se calcularon medidas de resumen de tendencia central y dispersión según la naturaleza de las variables y se organizaron en tablas de frecuencia; para el análisis bivariado y el establecimiento de las posibles asociaciones de halitosis con los posibles factores causales se aplicaron test de chi cuadrado y test exacto Fisher para variables categóricas y la prueba $t$ de Student para variables cuantitativas medidas en escala de razón. ${ }^{10}$

Para el análisis multivariado, se aplicó un análisis de regresión binomial, que estimó los RR (Riesgos Relativos: Razón de Prevalencia) y los intervalos de confianza del $95 \% .^{11,82}$ Se definio como variable dependiente halitosis a un palmo, a un metro y a tres metros y como posibles variables explicatorios, las caracteristicas sociodemográficas, factores generales y orales.

Para el análisis de regresión binomial bivariado inicial, se analizó la variable dependiente con cada una de las variables explicatorias en forma independiente y a partir de este, se seleccionaron para el modelo tinal las variables que obtuvieron una probabilidad igual o inferior a $\mathrm{p}=0.25$." Todo el análisis se realiz.o considerando un nivel de significancia $\alpha=0.05$.

Se siguieron las recomendaciones éticas establecidas en la resolución 008430 de 1993 del Ministerio de Salud de Colombia, según el titulo II en su articulo 11, numeral b que clasifica este estudio como de riesgo minimo, pues se trata de un estudio observacional de corte transversal, en el que se recolectaron y registraron los datos a través de procedimientos comunes consistentes en examen oral e interrogatorio al paciente.

\section{RESULTADOS}

\section{Análisis univariado}

Se evaluaron 102 sujetos de ambos géneros con promedio de edad $66.3+$ /-8.7años: rango de edad entre 50 y 80 años. Al categorizar la variable edad como menores de 70 y mayores a ésta. se observó que la mayor frecuencia de personas estaba en el rango de entre 50 y 69 años (55.9\%): el 69.6\% de los sujetos encuestados eran mujeres (Tabla 1). 


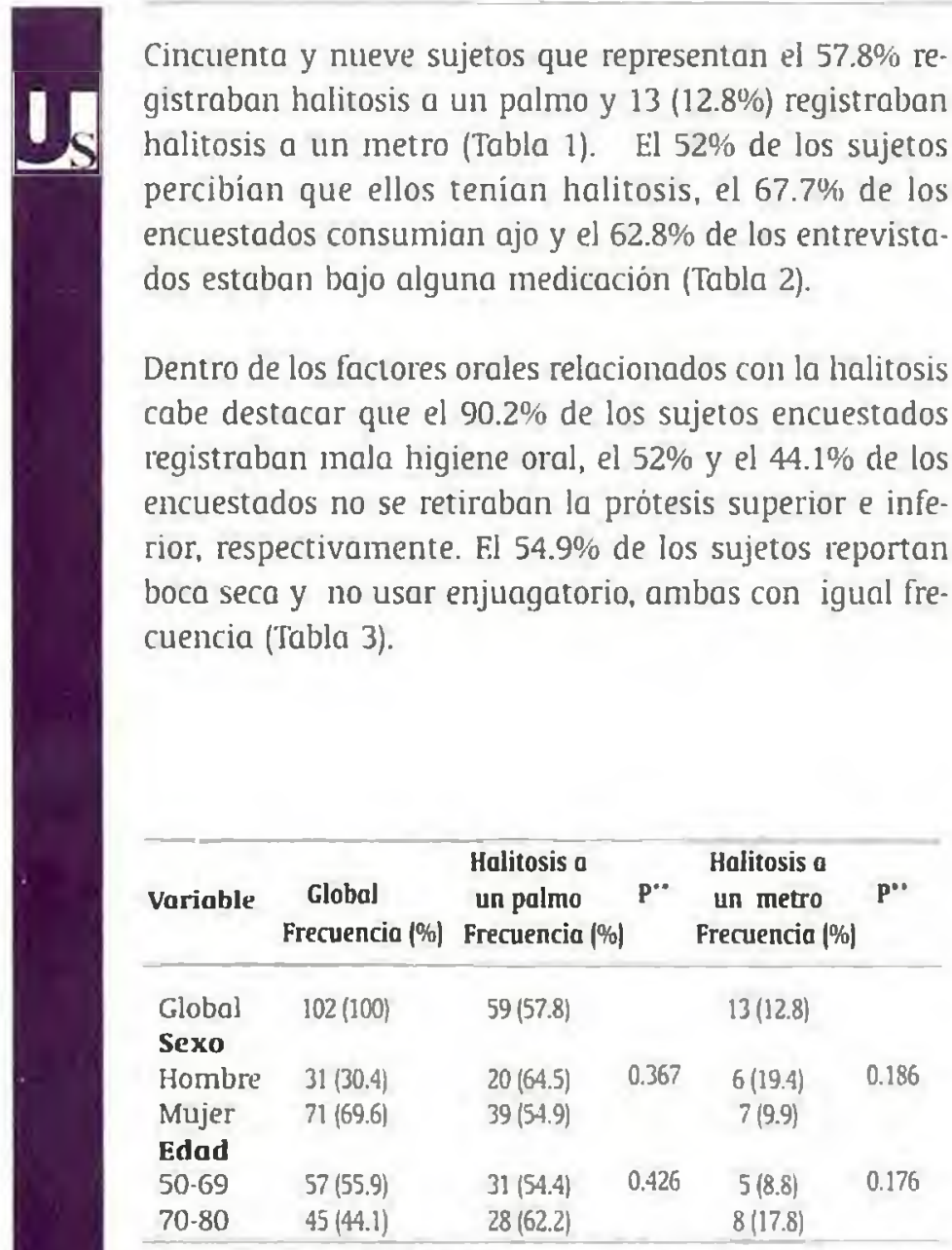

Tabla 1. Descripción global de la presencia de halitosis según las variables sociodemogróficas.

* Test de chi Cuadrado $\quad u=0.05$

\begin{tabular}{|c|c|c|c|}
\hline Variable & $\begin{array}{c}\text { Global } \\
N=102\end{array}$ & $\begin{array}{l}\text { Halitosis a } \\
\text { un palmo } \\
\text { Frecuencia (\%) }\end{array}$ & P.*. \\
\hline \multicolumn{4}{|l|}{$\begin{array}{l}\text { Factores relacionados con } \\
\text { Percepción }\end{array}$} \\
\hline Halitosis percibida por otros & $35(34.1)$ & $34(97.1)$ & $<0.001$ \\
\hline Halitosis outopercibida & 531 & $49(92.5)$ & $<0.001$ \\
\hline Prorege cuando habla & 29128 & $22(75.7)$ & $0.02^{* *}$ \\
\hline $\begin{array}{l}\text { Consulta por mal aliento } \\
\text { Factores relacionados con la } \\
\text { ingesta }\end{array}$ & $20(19.6)$ & $18(90)$ & 0.001 \\
\hline Consumo de ajo & $69(67.7)$ & $47(68.1)$ & $0.002^{\circ}$ \\
\hline $\begin{array}{l}\text { Frecuencia ingesta cuatro } \\
\text { comidas } \\
\text { Foctores médicos }\end{array}$ & $45(44.1)$ & $30(66.7)$ & 0.018 \\
\hline Medicación & $64(62.8)$ & $43(67.2)$ & $0.013^{\circ}$ \\
\hline
\end{tabular}

Tabla 2. Descripción de la presencia de halitosis a un palma según factores generales relacionados.

" Test de chi Cuadrado "."Test tixacto de Fisher $\quad u=0.05$

\section{Análisis bivariado}

Al analizar por variables sociodemográficas seguin el nivel de halitosis no se encontraron diferencias estadisticamente significativas al analizar la halitosis a un palmo y a un metro según sexo y edad (Tabla 1).

Al describir halitosis a un palmo segin los factores generales relacionados, se encontraron diferencias estadisticamente significativas para los criterios de halitosis percibida por otros y autopercibida $(\mathrm{p}<0.001)$ factores relacionados con la ingesta de ajo y con el consumo de cuatro comidas al día con $p=0.002$ y 0.018 , respectivamente y con factores relacionados con medicación $p=0.013$ ('Tabla 2).

Al describir halitosis a un palmo asociada con factores orales relacionados, se encontraron diferencias estadísticamente significativas para percepciones como sensación de boca seca, sensación de mal sabor en la boca y sabor salado, todas ellas con nivel $p<0.001$. Con igual nivel de significancia se encontraron diferencias para los criterios de cepillado de la lengua una vez al dia. lengua color café y cobertura gruesa de la lengua y también para los criterios retención de placa en la superficie externa de la base de la prótesis superior e inferior ('Tabla 3).

Al analizar halitosis a un metro segun los factores generales y orales relacionados, se encontraron diferencias estadisticamente significativas para los criterios orales relacionados con halitosis autopercibida, mal sabor en la boca y para sabor agrio, con niveles de significancia $p<0.001$. Para los factores generales relacionados con la halitosis se encontraron diferencias marginales para el criterio enfermedad cardiovascular $(p=0.076)$ (Tabla 4).

\section{Análisis multivariado}

El modelo de regresión binomial no ajustado, mostró que las criterios de percepciones relacionados con mal sabor en la boca con RR de 3.61; sabor agrio y salado con RR 3.4 y 3.8. respectivamente estaban asociados a halitosis a un palmo.

De igual forma se observó que nivel de placa en las superficies dentarias (RR 6.3), en la superficie externa de la base de prótesis inferior con nivel cubierta (RR 4.4) y medianamente cubierta (RR 6.3) se asaciaron significativamente con la presencia de halitosis a un palmo.

12

Ustasalud Odontología 2005; 4: 9-16 Rodriguez ML. y col 


\begin{tabular}{|c|c|c|c|}
\hline Variable & $\begin{array}{c}\text { Global } \\
N=102 \\
\text { Frecuencia }(\%)\end{array}$ & $\begin{array}{c}\text { Halitosis a } \\
\text { un palmo } \\
\text { Frecuencia }(\%)\end{array}$ & $\mathbf{p} \cdots$ \\
\hline \multicolumn{4}{|l|}{ Factores percepciones } \\
\hline Boca seca & $56(54.9)$ & $46(82.1)$ & $<0.001$ \\
\hline Mal sabor & $48(47.1)$ & $45(93.8)$ & $<0.001$ \\
\hline Sabor salado & $4(3.9)$ & $4(100)$ & $<0.001$ \\
\hline \multicolumn{4}{|l|}{ Factores de higiene de la prótesis } \\
\hline Cepillado una vez en el dia & $6(5.9)$ & $6(100)$ & 0.022 \\
\hline No usa enjuagatorio & $56(54.9)$ & $36(64.3)$ & 0.016 \\
\hline \multicolumn{4}{|l|}{ Factores relacionados con lengua } \\
\hline Cepillado una vez en el día & $21(20.6)$ & $18(85.7)$ & $<0.001$ \\
\hline Color caf $\dot{e}$ & $9(8.8)$ & $9(100)$ & $<0.001$ \\
\hline Cobertura gruesa & $24(23.5)$ & $23(95.8)$ & $<0.001$ \\
\hline \multicolumn{4}{|l|}{ Factores relacionados con la prótesis } \\
\hline No retira prótesis superior & $53(52)$ & $35(66)$ & $0.081^{\cdots 1-}$ \\
\hline No retira prótesis inferior & $45(44.1)$ & $31(68.9)$ & $0.045^{* *}$ \\
\hline \multicolumn{4}{|l|}{ Base de prótesis superior } \\
\hline Superficie externa totalmente cubierta & $28(27.4)$ & $22(78.6)$ & $<0.001$ \\
\hline $\begin{array}{l}\text { Superficie interna totalmente cubierta } \\
\text { Base de protesis inferior }\end{array}$ & $1(1)$ & $1(100)$ & 0.02 \\
\hline Superficie externa totalmente cubierta & $24(23.5)$ & $20(83.3)$ & $<0.001$ \\
\hline Superficie interna totalmente cubierta & $3(2.9)$ & $3(100)$ & 0.029 \\
\hline Higiene superficies dentales & $92(90.2)$ & $58(63)$ & 0.002 \\
\hline
\end{tabular}

Tabla 3. Descripción de la presencia de halitosis a un palmo según factores orales relacionados.

- Test de chi cuadrado - - Test Exactu de Fisher

+ +significancia marginal $\quad \alpha=0.05$

A nivel de la lengua cabe resaltar los criterios color café de la lengua (RR 4.4) y capa gruesa de la lengua con RR 4.1, se asociaron significativamente con la presencia de halitosis a un palmo (Tabla 5).

El modelo binomial ajustado mostró que las variables mal sabor en la boca (RR 2.16), sensación de boca seca (RR 1.48) y el consumo de ajo fueron los criterios que permanecieron como estadisticamente asociados con la presencia de halitosis a un palmo (Tabla 6).

El modelo de regresión binomial no ajustado para halitosis a un metro, permitió observar que las variables sensación de boca seca con RR 4.4, lengua color café con RR 21.1, lesiones en la mucosa labial con RR 8.8 y presencia de placa en la superficie interna de la base de la prótesis inferior se asociaron significativamente con este signo (Tabla 7).

El modelo final ajustado permite asociar significativamente la halitosis a un metro con lesiones en mucosa labial RR 7.8, lengua color café RR 19.6 y finalmente, con consumo de medicamentos con RR 2.95 (Tabla 8). No se registraron pacientes con halitosis a tres metros.

\section{DISCUSIÓN}

El análisis de la halitosis a un palmo en pacientes usuarios de prótesis totales que acuden a la consulta odontológica en las clínicas de la Universidad Santo Tomás mostró que mal sabor en la boca y la sensación de boca seca eran factores orales asociados con este fenó. meno; asi mismo las lesiones en mucosa labial, lengua color café podrian constituirse en factores bucales relacionados con la halitosis a un metro.

Kleinberg y Westbay, asociaron la halitosis a la reducción del flujo salival que ocurre al levantarse y se relaciona fundamentalmente con la reducción del suministro de oxigeno: $;^{13}$ afirman, además, que un rápido flujo de saliva aumenta el oxigeno disponible y disminuye la oportunidad de que los péptidos y las proteinas sean degradadas por las bacterias orales.

El mal aliento, es, una condición comủn, usualmente derivada de la actividad de la microbiota oral: hay evidencias que sugieren que las especies Gram negativas son las responsables de éste; ${ }^{\text {it }}$ tambiẻn, se ha observado que 


\begin{tabular}{|c|c|c|}
\hline Variable & $\begin{array}{l}\text { Halitosis a un metro } \\
\qquad \begin{array}{c}N=13 \\
\text { Frecuencia (\%) }\end{array}\end{array}$ & P** \\
\hline \multicolumn{3}{|l|}{ Factores percepciones } \\
\hline Halitosis percibida por otros & $9(69.2)$ & 0.01 \\
\hline Halitosis autopercibida & $13(100)$ & $<0.001$ \\
\hline Nivel débil de halitosis & $6(46.2)$ & $<0.001$ \\
\hline Antecedentes médicos & & \\
\hline $\begin{array}{l}\text { linfermedades cardiovasculares } \\
\text { Factores bucales }\end{array}$ & $7(53.9)$ & $0.076^{\cdots} \cdots$ \\
\hline Sensación de boca seca & $11(84.6)$ & $0.058^{\cdots}$ \\
\hline Mal sabor en la boca & $13(100)$ & $<0.001$ \\
\hline Sabor agrio en boca & $7(53.9)$ & $<0.001$ \\
\hline Cepillado prótesis, una vez al dia & $6(46.2)$ & $0.096^{\cdots}$ \\
\hline Lengua color café & $5(38.5)$ & 0.001 \\
\hline $\begin{array}{l}\text { Acumulación placa interior } \\
\text { Prótesis cubierta }\end{array}$ & $5(38.5)$ & $0.071^{\cdots}$ \\
\hline
\end{tabular}

Tabla 4. Descripcion de la presencia de halitosis a uл metro según foctones generales y urales relacionados.

"Test de chi Cuadrado "Test lixacto de Fisher $\quad u=0.05$

++ significancia marginal

las lesiones pigmentadas en lengua se asocian con la presencia de bacterias cromógenas, pues las condiciones locales y la composición bacteriana que habitan en la superficie de la lengua determinan el color que varía desde blanco o café claro hasta café oscuro o negro. ${ }^{15}$ Por lo tanto, se puede afirmar que el origen microbiano común de los dos fenómenos podria explicar la relación.

Yaegaki y Coil reportaran prevalencia de halitosis que alcanzaba niveles tan altos como del 50\% y que podría ser similar a los resultados referidos en este estudio. 16 Tapias, Duran y Galviz, en su estudio sobre los factores asociados a la prevalencia de halitosis reportaron una prevalencia de halitosis a un palmo de $24.6 \%$ y a un metro de $2.5 \%{ }^{17}$ la diferencia con lo reportado en la presente investigacion (prevalencia de $57.8 \%$ para halitosis a un palmo y $12.8 \%$ para halitosis a un metro) podria relacionarse con la población analizada, pues mientras Tapias y colaboradores trabajaron sobre grupos de personas mayores de 12 años, en esta investigación se consideraron a las personas con 50 años y más.

El tratamiento de la halitosis usualmente consiste en recomendar al paciente mejorar la higiene oral y el uso apropiado de enjuagatorios, pastas dentales, profilaxis dentales y limpieza de la lengua entre otros. ${ }^{1720}$ Estudios indican que el mal olor se deriva principalmente de la cobertura de la lengua, ${ }^{16.20}$ de tal forma que es más importante la limpieza de ésta, que el uso de enjuagues orales. Sin embargo, no se recomienda el uso de raspadores linguales o cepillos dentales para adultos para su limpieza por el posible daño que se puede ocasionar en esta superficie. Un cepillo dental pediátrico se constituye en la mejor alternativa para lograr una higiene lingual adecuada. ${ }^{16}$

La presente investigación mostró grandes depósitos de placa dentobacteriana en las superficies dentales y en las bases de prótesis, por lo que es necesario hacer énfasis en la higiene adecuada de las prótesis pues pueden constituirse en un factor asociado a la halitosis.

Fundamentados en los hallazgos de Tapias y colaboradores, se encontró comportamientos similares relacionados con el uso del cepillo dental una vez al día en el $6.6 \%$ de los sujetos, no usar enjuagatorio en el $54.9 \%$ de los encuestados y un comportamiento diferente en el consumo de ajo pues mientras en la citada investigación, el $26.2 \%$ de los sujetos consumian ajo en la presente, el consumo fue evidente en el $67.7 \%$ de los sujetos.

Sólo el $0.82 \%$ de la población encuestada por Tapias y colaboradores registraron lengua de color café, mientras que en el presente estudio fue evidente en el $38.5 \%$ de los sujetos encuestados. El $1.64 \%$ de los encuestados en la citada investigación registraron una cobertura gruesa de lengua, mientras que en esta fue evidente en $23.5 \%$ de las personas entrevistadas. En el citado estudio el nivel de mala higiene oral se observó en el 7.38\% de los sujetos y en el actual en el $90.2 \%$ de las personas investigadas. 'Tales diferencias podrian atribuirse a la edad y a los malos hábitos nutricionales y de higiene oral en los pacientes usuarios de prótesis.

Los aspectos relacionados con la percepción y autopercepción podrian estar estrechamente ligados a factores sicológicos. Los resultados registrados por Tanaka y colaboradores mostraron como aproximadamente la mitad de los pacientes con halitosis podrian clasificarse como pacientes con halitosis psicosomática y es importante para su tratamiento abordajes de tipo sicológico; 20 puntualiza además. que es probable que las mujeres tiendan a ser más ansiosas con respecto al mal aliento comparado con los hombres, aspectos que podrian estar repercutiendo en los resultados relacionados con las percepciones en la presente investigación. pues el $69.6 \%$ de la po. blación estaba constituida por mujeres.

Hay tres métodos para el análisis del mal aliento: la medición organoléptica, cromatografia de gases y el monitoreo de los sulfitos. La prueba organoléptica es 14 


\begin{tabular}{|c|c|c|c|}
\hline Variable & $\mathbf{R R}$ & IC $95 \%+t+$ & $\mathbf{P}$ \\
\hline \multicolumn{4}{|l|}{ Factores, percepciones } \\
\hline Sensación boca seca & 2.84 & $1.77-4.57$ & $<0.001$ \\
\hline Mal sabor en boca & 3.61 & $2.3-5.7$ & $<0.001$ \\
\hline Sabor agrio & 3.38 & $2.18-5.24$ & $<0.001$ \\
\hline Sabor salado & 3.76 & $2.45-5.79$ & $<0.001$ \\
\hline Sabor Metálico & 3.00 & $1.71-5.25$ & $<0.001$ \\
\hline Sabor dulzón & 2.88 & $1.55-5.32$ & 0.001 \\
\hline \multicolumn{4}{|l|}{ Factores de higiene } \\
\hline Cepillado de prótesis $>2 /$ dia & 0.46 & $0.35-0.6$ & $<0.001$ \\
\hline Cepillado de lengua $>2 /$ día & 0.34 & $0.19-0.63$ & 0.001 \\
\hline Índice dental de placa & 6.3 & $0.97-40.7$ & $0.053+$ \\
\hline Base ext prótesis inf media cubierta & 4.4 & $1.19-16.3$ & 0.026 \\
\hline Base ext de protesis inf cubierta & 6.3 & $1.7-23$ & 0.006 \\
\hline \multicolumn{4}{|l|}{ Factores lengua } \\
\hline Lengua color amarillo & 3.14 & $1.74-5.68$ & $<0.001$ \\
\hline Lengua color café & 4.41 & $2.49-7.82$ & $<0.001$ \\
\hline Grosor gruesa capa lengua & 4.07 & $1.72-9.63$ & 0.001 \\
\hline \multicolumn{4}{|l|}{ Factor prótesis } \\
\hline Retira prótesis inferior & 0.71 & $0.51-0.99$ & 0.044 \\
\hline \multicolumn{4}{|l|}{ Factores generales } \\
\hline Consumo ajo & 1.87 & $1.15-3.02$ & 0.01 \\
\hline Sinusitis & 1.82 & $1.54-2.15$ & $<0.001$ \\
\hline Enfermedades gástricas & 1.39 & $1.01-1.9$ & 0.04 \\
\hline Reflujo & 1.82 & $1.54-2.15$ & $<0.001$ \\
\hline
\end{tabular}

Tabla 5. Modelo de regresión binomial de halitosis a un palmo. Análisis Divariado.

++ Significancia marginal +++ intervalos de confianza, 95\% $\quad(x=0.05$

un examen sensorial fundamentado en las percepciones de un examinador. La cromatografia de gases es un equipo que consta fundamentalmente de un detector fotométrico especifico para detectar sulfuros en el aire de cavidad oral y es considerado como el gold standard para la medición de la halitosis, sin embargo, debido a las características de los equipos, se requiere de un operador experimentado para su manejo, lo cual genera dificultades durante su manipulación. ${ }^{\mathrm{it}}$

Se podria llegar a pensar que el uso de una prueba organoléptica podría sesgar los resultados. Sin embargo, se acepta que los seres humanos tienen un sentido del olfato capaz de detectar diferencias en la magnitud o en la con- centración de las moléculas olorosas que reduce esta posibilidad. ${ }^{19}$ Se sugiere que el análisis de reproducibilidad se lleve a cabo con dos o tres días de diferencia. ${ }^{16}$

El odontólogo debe considerar muchos factores relacionados con la higiene oral, las condiciones sistémicas y mentales antes de llegar a plantear un plan de tratamiento. ${ }^{30}$ Las necesidades de tratamiento deben estar siempre fundamentadas en un diagnóstico previo, donde se puedan determinar antecedentes y factores asociados. ${ }^{3}$

Los mecanismos de producción del mal olor son multifactoriales, y aunque no existe un factor único responsable, si existen condiciones especiales en los usuarios de

\begin{tabular}{lccc}
\hline \multicolumn{1}{c}{ Variable } & RR & IC 95\%+++ & P \\
\hline $\begin{array}{l}\text { Indice dental de placa } \\
\text { Base supf ext prótesis inf }\end{array}$ & 2.05 & $0.52-8.1$ & 0.304 \\
$\begin{array}{l}\text { media cubierta } \\
\begin{array}{l}\text { Base supf ext de prótesis inf } \\
\text { cubierta }\end{array}\end{array}$ & 2.88 & $0.86-9.63$ & $0.085++$ \\
$\begin{array}{l}\text { Mal sabor en boca } \\
\text { Sensación de boca seca }\end{array}$ & 3.31 & $0.99-11$ & $0.051++$ \\
Consume ajo & 2.16 & $1.36-3.41$ & $<0.001$ \\
\hline
\end{tabular}

Tabla 6. Modelo de regresión binomial final ajustado de halitosis a un palmo.

$$
++ \text { Significancia marginal } \quad+++ \text { Intervalos de confianza, } 95 \% \quad u=0.05
$$




\begin{tabular}{|c|c|c|c|}
\hline Variable & $\mathbf{R R}$ & $1095^{\prime \prime \prime}$ & $\mathbf{P}$ \\
\hline \multicolumn{4}{|l|}{ Factores orales } \\
\hline Sensación & 4.4 & $1.03-18.9$ & 0.045 \\
\hline Lengua color amarillo & 4.22 & $0.53-33.63$ & 0.174 \\
\hline Lengua color café & 21.1 & $2.8-159.06$ & 0.003 \\
\hline Lesión mucosa del labio & 8.8 & $5.17-15$ & 0.003 \\
\hline Retira prótesis inferior, dormir & 0.49 & $0.17 \cdot 1.41$ & 0.186 \\
\hline Base supf ext de prótesis inf. & 4.4 & 0.5932 .11 & 0.147 \\
\hline $\begin{array}{l}\text { Base supf int de prótesis inf. } \\
\text { Factores generales }\end{array}$ & 6 & $0.78-45.6$ & 0.083 \\
\hline Consumo cebulla & 3 & $0.7-12.8$ & 0.138 \\
\hline Erfermedades cardiovesculares & 2.43 & $0.88 \cdot 6.7$ & 0.083 \\
\hline EnfErmedades grásticas & 2.15 & 0.795 .9 & 0.133 \\
\hline Consumo medicamentos & 3.26 & $0.76-13.95$ & 0.110 \\
\hline
\end{tabular}

Tabla 7. Modelo de regresión binomial de halitosis a un metra. Anólisis dibariado.

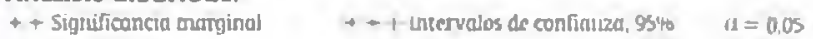

\begin{tabular}{lccl}
\hline \multicolumn{1}{c}{ Variable } & RR & IC95 $^{\ldots+\cdots}$ & P \\
\hline Factores orales & & & \\
Lesión mucosa labial & 7.84 & $3.7-16.7$ & $<0.001$ \\
Color amarillo lengua & 3.27 & $0.41-26.02$ & 0.261 \\
Color café de la lengua & 19.6 & $2.68-142.6$ & 0.003 \\
Factores generales & & & \\
Consumo medicamentos & 2.95 & $0.8-10.4$ & 0.092 \\
\hline
\end{tabular}

Tabla 8. Modelo de regresión binomial final ajustado de halitosis a un metro.

$$
\begin{aligned}
& ++ \text { Significancio marginal }+++ \text { Intervalos de confianza. 95\% } \\
& \alpha=0.05
\end{aligned}
$$

prótesis totales que hace necesario la identificación de factores particulares asociados a la halitosis y a la implementación de estrategias coherentes con las necesidades observadas en este grupo en particular con el propósito de brindarles una mejor calidad de vida.

\section{BIBLIOGRAFIA}

1. Kosenberg M. Kozlovsky A, Gelernter I. Cherniak O, Gavia J. Baht R. Eli I. Self estimation of oral malodor. J Dent Res 1995; 74: 1577 - 1582.

2. Eli l, Baht R, Koriat H, Rosenberg M. Self perception of breath odor. JADA 2001: 132: $621-626$.

3. Cuartas JC. Halitosis. Revista CES Odontologia 2003: 16: 83 - 88.

4. Marin DJ. Inlluencio de la boca en la calidad de vida relacionada con salud. UATREIA 2002: 15: $96-102$.

5. Mantilla S, Danser M. Sipos P, Rowshani B. Van der Velden U, Van der Weijden $F$ Cobertura de la lengua y cuentas bacterianas salivares en sujetos sanos y con gingivitis y en pacientes con periodontitis. Ustasalud 2002; 1: 7 - 17 .

6. República de Colombia. Ministerio de Salud. Fstudio Nacional de SaluL Bucal. lomo VII. Colombia 1999.
7. Pietrokovski J. Azuelos J, Tau S. Mostavoy R. Oral findings in elderly home resident in selected countries: Oral higiene conditions and ploque accumulation on denture surfaces. J Prosthet Dent 1995: 73: 136 - 141.

8. Microsoft Excel, 5.0, Microsoft Corporation, 1997

9. StataCorp. 2003. Stata Statistical Soft ware: Release 8.0. College Station, TX: Stata Corporation.

10. Pagano M. Gouvreau K. Principles of biostatistics. Duxbury press. Belmont, 1993.

11. Bautista I.E. Métodos avanzados de anälisis. Bucaramanga 2002.

12. Zochetti C. Consonni D. Bertazzi PA. Relationships between prevalence rote rotios and odds ratios in Cross- Sectional Studies. Int I Epidemiol 1997: 26: $220 \cdot 223$.

13. Kleinberg I. Westbay C. Salivary and metobolic fnctors involved in oral malodor formation. J. Periodontol 1992; 63: 768 - 775.

14. Sterer N, Bur-Ness Greenstein R. Rosenberg M. (-Galactosidase activity in saliva is Associated with oral malodor. J Dent Res 2002: 81: 182 - 185.

15. Regezi JA, Sciubba IJ. Patologia Bucal. Interamericana. McGraw- Hill, Mêxico, 1991. p.106

16. Yaegaki K. Coil JM. Examination, classification, and treatment of halitosis; Clinical perspectives. J Can Dent Assoc 2000; 66: 257 - 261.

17. Tapias E, Durán DC, Galviz S. Factores asociados a la prevalencia de halitosis en pacientes que acuden u las clinicas odontológicas de la Universidad Santo Tomás 2003 [Tesis de Grado]. Bucaramanga.

18. Tonzetich J. Production and origin of oral malodor: A review of mechanisms and methods of analysis. J Periodontol 1997; 48: 13-20.

19. Greenman J, DutTield J, Spencer P. Rosenberg M. Corry D. Saad S, Lenton P. Majerus G. Nachnani S, El-Maaytah M. Study on the organoleptic intensity scale for measuring ural malodor. J Dent Res 2004; 83: $81-85$.

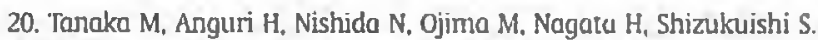
Reliability of clinical porameters for predicting the outcome of oral malodor treatment. J Dent Res 2003; 82: 518 - 522. 